\title{
Agreement between preoperative core needle biopsy and postoperative invasive breast cancer histopathology is not dependent on the amount of clinical material obtained
}

\author{
R O'Leary, K Hawkins, J C S Beazley, M R J Lansdown, A M Hanby
}

J Clin Pathol 2004;57:193-195. doi: 10.1136/jcp.2003.12914

\begin{abstract}
Aims: To establish the relation between the amount of breast core needle biopsy (CNB) material examined and agreement between preoperative and postoperative histopathology parameters in invasive breast cancer.

Methods: The CNB and surgical specimen histopathology reports of 113 patients with invasive breast carcinoma were reviewed and the total amount of CNB material examined for each case was determined. Agreement was calculated for tumour type, grade, mitoses, nuclear pleomorphism, and tubule formation. Associations between the amount of CNB material and histopathology agreement before and after surgery were explored using binary logistic regression.

Results: Tumour type and grade agreed in $65.4 \%$ and $61.6 \%$ of cases, respectively. The components used to calculate grade-nuclear pleomorphism (57.4\%), mitoses (59.4\%), and tubule formation (55.6\%)-agreed slightly less frequently. The proportion of cases with preoperative and postoperative assessments that agreed did not depend on the number of cores collected or the total amount of material examined.

Conclusion: Neither tumour type and grade, nor the individual components used to calculate grade agreed consistently between the CNB and surgical specimen. The number of cores collected and the total amount of material reviewed by the pathologist does not influence the likelihood of agreement between preoperative and postoperative histopathology reports.
\end{abstract}

B reast cancer tumour sampling by core needle biopsy (CNB) is widely used in many centres and has proved to be up to $97 \%$ accurate at confirming the presence of malignant tissue within a suspicious lesion. ${ }^{12}$ It has been established previously that CNBs generally underestimate tumour grade and can also incorrectly type tumours (for review see Denley and colleagues ${ }^{3}$ ). However, to our knowledge, there are no studies that have investigated whether the amount of material available to the pathologist from the CNB influences the agreement between the preoperative and postoperative histopathology of invasive disease.

Detailed preoperative histopathological information is increasingly required from pathologists and is potentially useful in determining the treatment of patients who may benefit from neoadjuvant chemotherapy, and also in the development of preoperative prognostic models. The ability to determine accurately tumour type and grade and therefore the individual components of the grade-tubule formation, mitoses, and nuclear pleomorphism-is crucial in the development of any preoperative prognostic tool. It seems probable that there is a correlation between the amount of clinical material obtained from the CNB and its accuracy. Therefore, we explored the relation between the amount of clinical material available for examination by the pathologist and how faithfully preoperative histopathology reflects that of the surgical specimen.

"Detailed preoperative histopathological information is
increasingly required from pathologists"

\section{PATIENTS AND METHODS}

The pathology records at our centre from 1999 to 2002 were reviewed to identify those patients who had undergone CNB for a suspicious breast lesion. Patients were included in our study if both their CNB and surgical specimen reports were unequivocal in describing the presence of invasive breast carcinoma. Patients who had previously received, or were receiving, chemotherapy were excluded, and 113 patients who had undergone CNB (all Tru-cut l biopsy needle) and subsequent primary surgical excision for breast carcinoma were identified. All patients had been managed in accordance with the UK National Health Service (NHS) breast screening programme guidelines, which require surgical excision within two weeks of diagnosis. ${ }^{4}$ The original haematoxylin and eosin stained CNB slides were retrieved and reviewed by a consultant histopathologist, and we collected the following information from the histopathology reports: anonymised patient identification number, type according to the UK NHS breast screening programme guidelines, ${ }^{4}$ grade, nuclear pleomorphism, mitoses, and tubule formation according Elston and Ellis's modification of the Bloom and Richardson system. ${ }^{5}$

Core length was measured manually using a ruler, but to ensure the accuracy of this method at detecting and measuring all of the biopsy material on the slide we took a random sample of $10 \%$ of the biopsies and measured these using the Nikon Ellipse E-1000 microscope/Lucia image analysis system (Nikon, Kingston upon Thames, UK). No significant difference between the two methods of measurement was found ( $\mathrm{p}=0.42 \mathrm{l}$, paired $t$ test). A "core index" for each patient was calculated, which was derived by multiplying the number of cores taken by the number of levels viewed by the histopathologist by the total length of the cores. For example, for three levels of three cores measuring $23 \mathrm{~mm}$ in total the core index would be 207 . Repeated levels were occasionally requested for cores where there was difficulty in confirming the diagnosis. Potentially, this could confound our analysis, so that cases where only the

Abbreviations: CNB, core needle biopsy; NHS, National Health Service 
standard three levels were available $(n=110)$ were examined as a separate dataset. Binary logistic regression (using Minitab ${ }^{\mathrm{TM}}$ Statistical Software 13.1; Minitab Inc, State College, Pennsylvania, USA) was used to assess our hypothesis that the proportion of preoperative and postoperative assessments that agree may be a function of the number of CNBs collected or the total amount of clinical material obtained. Two models, one containing the number of cores and one with the core index as an independent variable, were investigated for each histopathology variable. Independent variables were assessed at the $10 \%$ level of significance.

\section{RESULTS}

Agreement between the CNB and the surgical biopsy specimen for grade, mitoses, tubules, pleomorphism, and type in 113 patients with breast cancer was assessed. Tumour type agreed in $65.41 \%$ of cases, which was similar to grade $(61.65 \%)$. The components used to calculate grade-nuclear pleomorphism $(57.40 \%)$, mitoses $(59.40 \%)$, and tubule formation (55.64\%)-agreed less frequently. Grade and mitoses were more likely to be underscored preoperatively than overscored; grade was underscored preoperatively in $24.06 \%$ of cases compared with $14.28 \%$ overscored, and mitoses were underscored in $33.08 \%$ of cases compared with $7.52 \%$ overscored. Tubule formation was more likely to be overscored preoperatively $(12.78 \%)$ than postoperatively (31.58\%). Pleomorphism was overscored and underscored equally frequently $(21.05 \% \vee 21.81 \%)$.

Table 1 shows the assessment of agreement between preoperative and postoperative assessments for tumour type, grade, mitoses, nuclear pleomorphism, and tubules. The number of cores did not influence the proportion of cases with agreement between CNB and postoperative histopathology $(p=0.42$, binary logistic regression). The more sophisticated measure of the amount of clinical material viewed by the pathologist, the core index, was used to investigate further the influence of clinical material on agreement. This was calculated from the number of cores, total length, and number of levels viewed, and was therefore effectively an index of the information available to the pathologist. Again, using binary logistic regression, there was no significant association between the amount of biopsy material viewed and the proportion of preoperative and postoperative histopathology agreements. We were concerned that our results could be compromised by the cases where repeated levels were requested. Therefore, we examined the 110 "standard" cases where only levels 1-3 were viewed as a separate dataset. Again, no significant difference was found in either the mean number of cores or the mean core indices for agreement between the parameters (all p values $>0.1$, binary logistic regression).

The means, medians, ranges, and distributions of core indexes for grade and tumour type were, in fact, almost identical between the two groups shown in fig 1 , which describes the full dataset of 113 patients. The distributions of core indexes for mitoses, pleomorphism, and tubule formation are not shown, but were broadly similar, as would be expected from the results for tumour grade.

\section{DISCUSSION}

Accurate preoperative assessment of breast cancer is becoming increasingly important. Surgeons and pathologists are looking beyond the ability to diagnose invasive disease accurately to tools that will allow the earlier selection of patients for adjuvant chemotherapy or for more conservative surgical procedures.

We have assessed tumour grade, tumour type, and the components used to calculate grade. We used the grade components for two reasons: (1) if grade did not frequently agree with postoperative histology we wanted to establish whether one particular component contributed disproportionately to the disagreement; and (2) perhaps more importantly, we were interested to see whether we could identify one component that was consistently accurate, and which would therefore make a more suitable candidate for preoperative prognosis.

Broadly, we found that all of the parameters we assessed agreed between preoperative and postoperative histopathology in $55-65 \%$ of cases. This is consistent with two similar studies. ${ }^{67}$ However Harris et al reported significantly higher rates of agreement for tubule formation and pleomorphism than was seen in our study. ${ }^{6}$ Where there was disagreement, it was more likely to be underscored preoperatively than overscored, which is a well recognised phenomenon. ${ }^{3}$ As other authorities have proposed, it is probable that the grading of the surgical specimen, which (by necessity) relies on finding the worst areas to score the grade components, will generally grade higher than a CNB, which samples a very small fraction of typically heterogeneous tumours.

\begin{tabular}{|c|c|c|c|c|c|c|c|}
\hline & \multicolumn{4}{|c|}{ Number of cores } & \multicolumn{3}{|c|}{ Core index } \\
\hline & $\mathrm{N}$ & Median & Range & $\mathrm{p}$ Value & Median & Range & p Value \\
\hline \multicolumn{8}{|l|}{ Type } \\
\hline Agree & 78 & 3 & $1-7$ & \multirow[t]{2}{*}{0.415} & 161 & $15-1260$ & \multirow[t]{2}{*}{0.331} \\
\hline Disagree & 35 & 2 & $1-5$ & & 144 & $20-760$ & \\
\hline \multicolumn{8}{|l|}{ Grade } \\
\hline Agree & 67 & 2 & $1-5$ & \multirow{2}{*}{0.172} & 153 & $20-812$ & \multirow[t]{2}{*}{0.190} \\
\hline Disagree & 46 & 3 & $1-7$ & & 151 & $15-1260$ & \\
\hline \multicolumn{8}{|l|}{ Mitoses } \\
\hline Agree & 66 & 2 & $1-4$ & \multirow[t]{2}{*}{0.300} & 145.5 & $20-812$ & \multirow[t]{2}{*}{0.148} \\
\hline Disagree & 47 & 3 & $1-7$ & & 171 & $15-1260$ & \\
\hline \multicolumn{8}{|c|}{ Tubule formation } \\
\hline Agree & 60 & 3 & $1-7$ & \multirow{2}{*}{0.738} & 145.5 & $15-1260$ & \multirow{2}{*}{0.983} \\
\hline Disagree & 53 & 2 & $1-5$ & & 160 & $20-812$ & \\
\hline \multicolumn{8}{|c|}{ Pleomorphism } \\
\hline Agree & 64 & 3 & $1-7$ & \multirow[t]{2}{*}{0.615} & 152 & $42-1260$ & \multirow[t]{2}{*}{0.530} \\
\hline Disagree & 49 & 2 & $1-4$ & & 153 & $15-660$ & \\
\hline
\end{tabular}

In total, 113 patients were examined to find out whether the number of cores collected during the diagnosis of a suspicious lesion correlated with the likelihood of agreement between preoperative and postoperative histology. This was assessed by two methods: (1) a count of the number of cores and (2) an index of the amount of material examined by the pathologist (as described in Patients and methods). Mitoses, tubule formation, and nuclear pleomorphism were components of tumour grade. The $\mathrm{p}$ value was determined by binary logistic regression. 

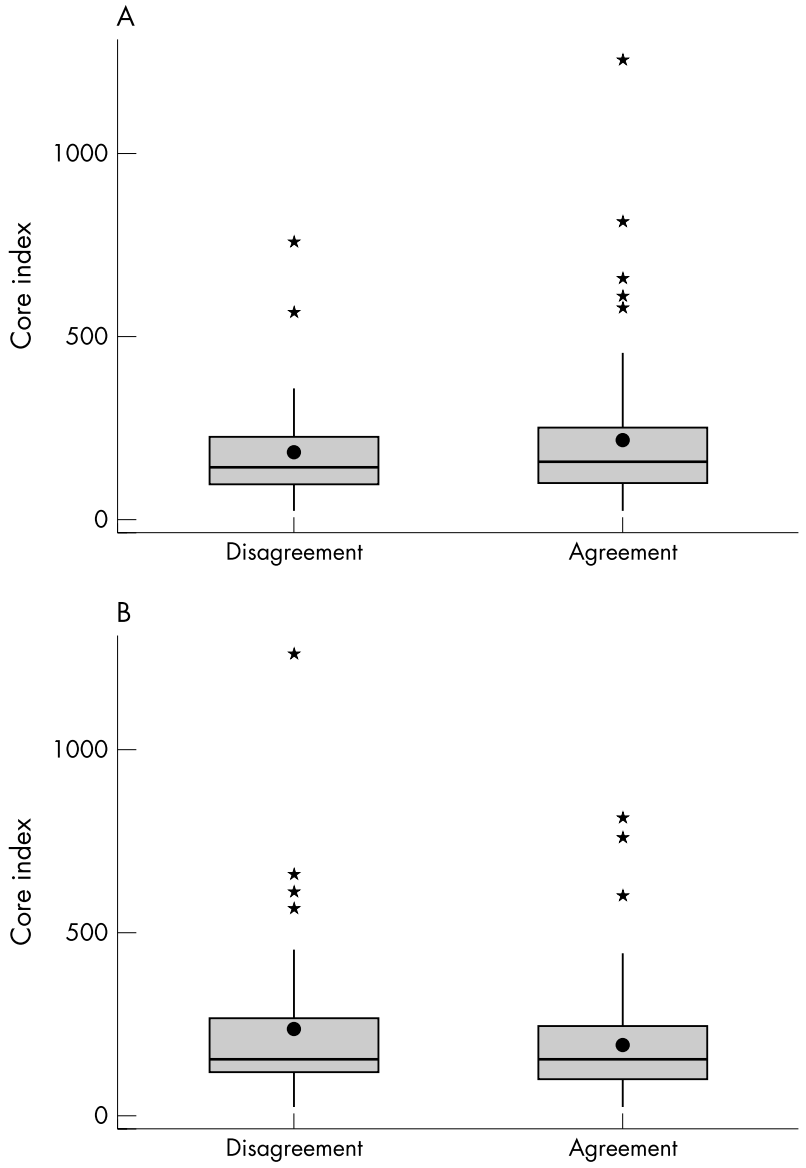

Figure 1 Box plots of core indexes for cases where there was agreement or disagreement between preoperative and postoperative histopathology. In a dataset of 113 patients, the core index was calculated (as described in Patients and methods) to describe the total amount of material examined by the pathologist during reporting of the needle core biopsy. The core index is shown for cases where there was agreement or disagreement between preoperative and postoperative histology for (A) grade and (B) tumour type. Boxes show median values and the interquartile ranges, the whiskers show the ranges and the outliers are indicated by the asterisks; the means (solid circles) are shown for comparison.

We have asked a fundamentally different question to previous work examining the relation between the number of cores and diagnosis. Typically, these studies have sought to determine the relation between CNB number and diagnostic accuracy. ${ }^{8}{ }^{9}$ We were interested to find out whether, in cases where there is a definite diagnosis, the amount of clinical material available influences agreement between CNB and the surgical specimen.

\section{"This surprising and somewhat paradoxical result defies} simple explanation"

We could find no significant association between either the number of cores collected or the total amount of material viewed by the pathologist and the likelihood of agreement. This surprising and somewhat paradoxical result defies simple explanation. It would be expected that the more

\section{Take home message}

- In cases of invasive breast carcinoma, the number of cores and the amount of material viewed by the pathologist does not influence agreement between the histopathology reported from the preoperative core biopsy and the definitive excision specimen

material sampled from a tumour the better the representation and therefore agreement. One of many possible explanations is that doubling or even tripling of the number or length of a typical CNB only represents single per cent sampling increases from the total volume of even a $\mathrm{l} \mathrm{cm}^{3}$ tumour.

However, these data do suggest that provided there is sufficient $\mathrm{CNB}$ to confirm the presence of invasive disease, there is little to be gained from further biopsies in terms of both accurate staging of grade and tumour type and also improving the quality of information available for putative prognostic tools. Moreover, we show that confidence in the CNB data to provide prognostic information does not increase with larger or more biopsies.

\section{Authors' affiliations}

R O'Leary, J C S Beazley, School of Medicine, University of Leeds, Leeds, LS2 9NL, UK

K Hawkins, Clinical Trials and Research Unit, University of Leeds, 17 Springfield Mount, Leeds, LS2 NG, UK

M R J Lansdown, Breast Unit, St James's University Hospital, Leeds, LS9 TF, UK

A M Hanby, Department of Histopathology, St James's University Hospital

Correspondence to: Professor A M Hanby, Department of Pathology, St James's University Hospital, Leeds, LS9 F, UK;

andrewh@pathology.leeds.ac.uk

Accepted for publication 19 August 2003

\section{REFERENCES}

1 Pinder SE, Elston CW, Ellis IO. The role of pre-operative diagnosis in breast cancer. Histopathology 1996;28:563-6.

2 Verkooijen HM, Peeters PHM, Buskens E, et al. Diagnostic accuracy of largecore needle biopsy for nonpalpable breast disease: a meta-analysis. $\mathrm{Br} J$ Cancer 2000;82:1017-21.

3 Denley H, Pinder SE, Elston CW, et al. Preoperative assessment of prognostic factors in breast cancer. J Clin Pathol 2001;54:20-4.

4 Non-operative Diagnosis Subgroup of the National Coordinating Group for Breast Screening Pathology. Guidelines for non-operative diagnostic procedures and reporting in breast cancer screening. Sheffield: NHS Cancer Screening Programmes, 2001 (NHS Publication No. 50, ISBN 187199744 5).

5 Elston CW, Ellis IO. Pathological prognostic factors in breast cancer. I. The value of histological grade in breast cancer: experience from a large study with long term follow-up, Histopathology 1991;19:403-10.

6 Harris GC, Denley HE, Pinder SE, et al. Correlation of histologic prognostic factors in core biopsies and therapeutic excisions of invasive breast carcinoma. Am J Surg Pathol 2003;27:11-15.

7 Sharifi S, Peterson MK, Baum AK, et al. Assessment of pathologic prognostic factors in breast core needle biopsies. Mod Pathol 1999;12:941-5.

8 Liberman L, Dershaw DD, Rosen PP, et al. Stereotaxic 14-gauge breast biopsy-how many core biopsy specimens are needed. Radiology 1994;192:793-5.

9 Fishman JE, Milikowski C, Ramsinghani R, et al. US-guided core-needle biopsy of the breast: how many specimens are necessary? Radiology 2003;226:779-82. 